\title{
Pharmacokinetics, Safety and Tolerability of Bencycloquidium Bromide, a Novel Selective Muscarinic M1/M3 Receptor Antagonist, After Single and Multiple Intranasal Doses in Healthy Chinese Subjects
}

\section{An Open-Label, Single-Center, First-in-Human Study}

Luning Sun, ${ }^{1}$ Li Ding, ${ }^{1}$ Yongqing Wang, ${ }^{2}$ Wenjia Zhou, ${ }^{1}$ Zhengyu Yan, ${ }^{1}$ Weilin Sun, ${ }^{2}$ Hongwen Zhang, ${ }^{2}$ Ning $\mathrm{Ou}^{2}$ and Xiaoping Chen ${ }^{3}$

1 China Pharmaceutical University, Nanjing, China

2 First Affiliated Hospital of Nanjing Medical University, Nanjing, China

3 Beijing Shiqiao Biological and Pharmaceutical Co. Ltd, Beijing, China

\section{Abstract}

Background: Bencycloquidium bromide (BCQB) is a novel, potent and selective muscarinic M1/M3 receptor antagonist under development for the treatment of rhinorrhea in rhinitis. The pharmacokinetics and safety of BCQB in animals have been established in preclinical studies. However, no clinical pharmacokinetic data are available for BCQB in humans.

Objective: The aim of this first-in-human study was to evaluate the pharmacokinetics, safety and tolerability of BCQB following single and multiple intranasal doses in healthy Chinese subjects.

Methods: The clinical trial was comprised of the following four studies: (i) an open-label, single-dose escalation study to evaluate the safety and tolerability in healthy subjects after intranasal doses of BCQB ranging from 45 to $450 \mu \mathrm{g}$ (total of six doses); (ii) an open-label, multiple-dose escalation study to assess the safety and tolerability in healthy subjects after intranasal administration with 120 and $150 \mu \mathrm{g}$ doses of BCQB (360 and $450 \mu \mathrm{g} / \mathrm{day}$ ) administered three times daily for 15 days; (iii) a randomized, open-label and parallel-group design to evaluate the single-dose pharmacokinetics of BCQB after intranasal dosing (45, 90, and $180 \mu \mathrm{g}$ ); and (iv) ten subjects received $120 \mu \mathrm{g}$ of BCQB by intranasal administration, three times daily for 5 days with a final single dose on day 7 to assess its multiple-dose pharmacokinetics. Safety and tolerability of BCQB were evaluated by monitoring adverse events (AEs), ECG 
recordings, vital signs and clinical laboratory parameters. The pharmacokinetic parameters for BCQB were calculated by software using noncompartmental methods.

Results: All AEs were mild, of limited duration and no more frequent at higher doses. There was no serious adverse event, death or withdrawal. No clinically significant change was noted in clinical laboratory parameters, cardiac parameters or vital signs. Following single intranasal dosing, BCQB was rapidly absorbed with a median time to maximum concentration $\left(\mathrm{t}_{\max }\right)$ of 8 minutes for 45,90 , and $180 \mu \mathrm{g}$ dose groups; the plasma concentration of BCQB decreased in a biphasic manner with the mean half-life $\left(\mathrm{t}_{1 / 2}\right)$ of 8.5 hours; the maximum concentration $\left(\mathrm{C}_{\max }\right)$ and area under the plasma concentration-time curve (AUC) of BCQB increased linearly across the examined dose range of $45-180 \mu \mathrm{g}$. During the multiple dosing, the steady state was achieved within 3 days of $120 \mu \mathrm{g}$ three times daily dosing of BCQB. A slightly greater AUC was observed after 5 days of multiple dosing, with the mean accumulation ratio of 1.26; however, the half-life was unchanged.

Conclusion: BCQB was safe and well tolerated in healthy Chinese subjects when administered intranasally with single and multiple doses across the doses studied. The mean $\mathrm{C}_{\max }$ and AUC increased proportionally to the studied doses, and the steady state was achieved within 3 days after three times daily dosing. A slight accumulation of BCQB following multiple dosing was observed. The pharmacokinetics, safety and tolerability profiles of BCQB pose it as a good candidate for further development in the treatment of rhinorrhea in rhinitis.

\section{Introduction}

Bencycloquidium bromide, 3-\{(2-cyclopentyl2-hydroxy-2-phenyl) ethoxy\}-1-methyl-1-azabicyclo $[2,2,2]$ octane bromide (BCQB, figure 1$)$, is a novel selective muscarinic M1/M3 receptor antagonist for the treatment of rhinorrhea in rhinitis by intranasal administration. Rhinitis, an inflammation of the nasal mucous membrane, is one of the most common diseases, and is estimated to affect $10-40 \%$ of the global population with increasing prevalence in both children and adults. ${ }^{[1,2]}$ Currently, ipratropium bromide (IB) is the only muscarinic antagonist in clinical use for the treatment of rhinorrhea in rhinitis. ${ }^{[3]}$ However, the anticholinergic effect of IB is short-acting, and IB is less selective among the M1, M2, and M3 muscarinic receptors. ${ }^{[4]}$ Recently, long-term use of inhaled IB has been shown to be associated with an increased risk of adverse cardiovascular outcomes in patients, ${ }^{[5]}$ which may be related to its action on the muscarinic M2 receptor in the heart. Given the high prevalence of rhinitis and the undesirable safety profile of IB, the development of additional options is clearly warranted. Many studies have shown that intranasal BCQB has good efficacy in the treatment of rhinitis especially rhinorrhea in preclinical studies. ${ }^{[6-10]}$ Additionally, BCQB displayed a better safety profile than IB

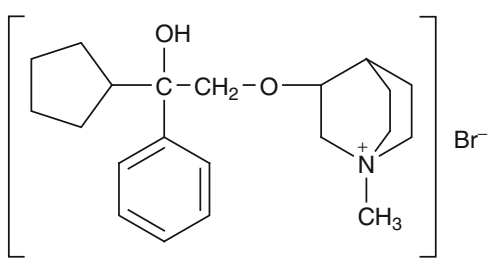

Fig. 1. Chemical structure of bencycloquidium bromide. 
due to its high selectivity for the M1 and M3 receptors over the M2 receptor. ${ }^{[11,12]}$ As a result, M2 cardiac receptors are spared thereby reducing the risks of cardiovascular adverse events. ${ }^{[13]}$ Preclinical toxicity studies also showed no apparent change in the ECG or heart rate in $\operatorname{dogs}^{[13]}$ and rats. ${ }^{[14]}$ Our recent phase II clinical trial in China showed that intranasal administration of $\mathrm{BCQB}$ was effective in reducing rhinorrhea with few side effects. Preclinical studies described the pharmacokinetics, tissue distribution, excretion and metabolism of BCQB after intranasal dosing in rats ${ }^{[15-18]}$ or beagle dogs. ${ }^{[19]}$ However, no data are available on the pharmacokinetics, safety and tolerability of BCQB in humans. Therefore, as a first-in-human (FIH) clinical trial, this study was conducted to evaluate the safety, tolerability and pharmacokinetics of BCQB after single and multiple intranasal doses in healthy Chinese subjects.

\section{Methods}

The FIH clinical trial was performed at a single center (First Affiliated Hospital of Nanjing Medical University) in Nanjing, China. The study was approved by the Ethics Committee at this study center and was conducted in accordance with guidelines for the Declaration of Helsinki and Good Clinical Practice (GCP) in China. All subjects were informed of the investigational nature of this study, and signed an informed consent statement prior to the initiation of the study.

\section{Subjects}

All eligible subjects were men or women aged 20-50 years, and were of Chinese origin (table I). Subjects' health states were analyzed on the basis of medical history, physical examination, eye examination, laboratory examination, and ECG.

The following exclusion criteria were applied for subjects in this clinical trial: a history of clinically significant cardiovascular, renal, urinary tract, hepatic, pulmonary, gastrointestinal, eye, mouth, nose, or mucosal diseases that might interfere with absorption, distribution, metabolism, or excretion of BCQB; a history of nervous system

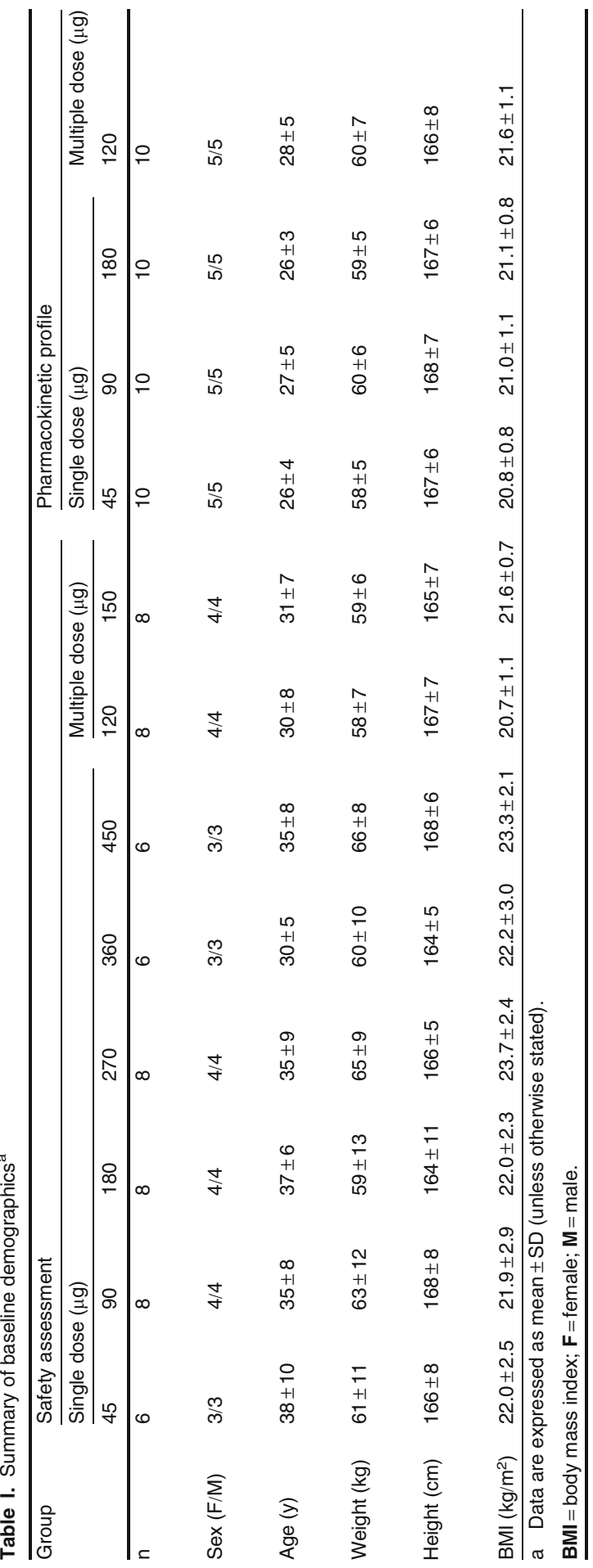


or muscle disease, seizure disorder or a psychiatric disorder that might hinder compliance with the study; a history of known allergy or intolerance to any drugs; a history of tobacco, alcohol or drug abuse; those with abnormalities in clinical laboratory parameters; those who had received an investigational drug, or donation of blood in the preceding 3 months, or had received any drug within 2 weeks before the study start date, or was considered by the investigator, for any reason, to be an unsuitable candidate for receiving BCQB. Females who were lactating or who had a positive pregnancy test were also ineligible.

\section{Study Drug and Administration}

BCQB nasal sprays used in these studies were manufactured by Beijing Shiqiao Biological and Pharmaceutical Co. Ltd (Beijing, China). The intranasal formulation provided different doses $(22.5,45,60,75,90,135,180$, and $225 \mu \mathrm{g})$ of BCQB in a $0.09 \mathrm{~mL}$ spray from a single-dose metered sprayer. The same metered sprayer $(0.09 \mathrm{~mL} /$ spray $)$ with different drug loads was used in tolerability and pharmacokinetic studies. For intranasal administration, each subject received a single spray in each nostril, for a total of two sprays. For example, the dosage of $45 \mu \mathrm{g}$ was provided by a spray of $22.5 \mu \mathrm{g}$ /spray in each nostril $(22.5 \mu \mathrm{g} /$ spray $\times 2)$. Prior to the administration of $\mathrm{BCQB}$, the subject gently blew his or her nose. A physician administered the nasal spray and attempted to concentrate the application on the lateral nasal wall, particularly along the inferior and middle turbinate mucosa, according to the standard operating procedures (SOPs).

\section{Study Design}

\section{Single-Dose Escalation Tolerability Study}

An open-label, single-dose escalation design was used to evaluate the safety and tolerability of BCQB after intranasal dosing (see table II). Subjects, $50 \%$ male and $50 \%$ female, were subsequently enrolled into the $45,90,180,270,360$, and $450 \mu \mathrm{g}$ dose groups $(6-8$ subjects in each

Table II. Study design

\begin{tabular}{lcc}
\hline Period & Number ${ }^{\text {a }}$ & Dose groups $(\mu \mathrm{g})$ \\
\hline Period $1^{\text {b,c }}$ & 6 & 45 \\
Single-dose escalation tolerability study & 8 & 90 \\
& 8 & 270 \\
& 8 & 360 \\
& 6 & 450 \\
Period $2^{\text {b,c,d }}$ & 6 & 120 tid for 14 days (360 $\mu \mathrm{g} /$ day) \\
Multiple-dose escalation tolerability study & 8 & 150 tid for 14 days (450 $\mu \mathrm{g} /$ day) \\
Period $3^{\mathrm{e}}$ & 8 & 45 \\
Single-dose pharmacokinetic study & 10 & 90 \\
& 10 & 180 \\
Period $4^{f}$ & 10 & 120 tid for 5 days \\
Multiple-dose pharmacokinetic study & 10 & \\
\hline a The number of subjects in each dose group. & & \\
b An open-label, dose escalation design was used. & & \\
c The trial would not proceed to the higher dose group until the safety and tolerability of the lower dose group were confirmed. \\
d The trial began with the 120 $\mu$ g dose group (360 $\mu \mathrm{g} /$ day), and this dosage was assigned according to the results of the single-dose \\
e tolerability study.
\end{tabular}


group). The trial was designed to begin with the $45 \mu \mathrm{g}$ dose group and would not proceed to the higher dose group until the safety and tolerability of the lower dose group was confirmed.

\section{Multiple-Dose Escalation Tolerability Study}

An open-label, multiple-dose escalation design was performed to begin with the $120 \mu \mathrm{g}$ dose group $(360 \mu \mathrm{g} /$ day $)$ according to the results of the singledose tolerability study and would not proceed to the higher dose group $(450 \mu \mathrm{g} /$ day $)$ until the safety and tolerability of the $360 \mu \mathrm{g}$ dose group was confirmed (see table II). Subjects, 50\% male and $50 \%$ female, were also subsequently enrolled into two dose groups (eight subjects in each), and were given $120 \mu \mathrm{g}(360 \mu \mathrm{g} / \mathrm{day})$ or $150 \mu \mathrm{g}(450 \mu \mathrm{g} / \mathrm{day})$ of BCQB via nasal spray three times daily (at $7.30 \mathrm{am}, 12: 00 \mathrm{pm}$ and $7: 00 \mathrm{pm}$ ) for 14 days to assess its safety and tolerability.

\section{Pharmacokinetic Study}

A randomized, open-label and parallel-group design was used to evaluate the pharmacokinetic profile of BCQB after single intranasal dosing (see table II). Thirty healthy subjects, $50 \%$ male and $50 \%$ female, were randomized into 45,90 , and $180 \mu \mathrm{g}$ dose groups (ten subjects in each) for the determination of the pharmacokinetic profile of a single-dose BCQB by the investigator. Another ten subjects, $50 \%$ male and $50 \%$ female, were administrated $120 \mu \mathrm{g}$ of BCQB by intranasal sprays on day 1 ; received no treatment on day 2 ; and continued to receive the study drug three times daily (at 7:30am, 12:00pm and 7:00pm) from days 3 through 7 to assess multiple-dose pharmacokinetics (see table II).

The subjects were required to fast overnight (12 hours) before administration, while standard meals and water intake were provided 2 hours postdose. Blood samples $(5 \mathrm{~mL})$ were collected at 0 hours (pre-dose), 2, 5, 10, 15, 30 minutes, 1, 2, 3, $5,7,12,24$, and 48 hours post-dose for the singledose study. For the multiple-dose study, blood samples $(5 \mathrm{~mL})$ were collected prior to dosing on days $1,5,6$, and 7 ( 0 hours prior to dosing) and 2, $5,10,15,30$ minutes, $1,2,3,5,7,9,12,15,24$, and 36 hours post-dose on day 1 and day 7. Plasma was separated and stored at $-20^{\circ} \mathrm{C}$ for analysis.
Urine samples were collected at 0 hours (pre-dose), $0-2,2-4,4-6,6-8,8-10,10-12,12-24,24-36$, and 36-48 hours post-dose for the single-dose study. The total volume of urine in each time interval was recorded and stored at $-20^{\circ} \mathrm{C}$ for analysis.

\section{Safety Monitoring}

Throughout the study, all subjects remained in the study unit under continuous observation. Details of adverse events (AEs) were obtained and recorded by the study physicians. Routine safety and tolerability were evaluated through AE reporting by the investigators and subjects, on the basis of vital signs, physical examination, laboratory examination (routine blood, urine and feces test, occult blood test and blood biochemical test) and ECG, which were performed at scheduled intervals during the studies. AEs that occurred during the study were classified as mild (awareness of a sign or symptom but comfortably tolerated), moderate (discomfort that may interfere with daily activities) or serious (death, life-threatening, requiring hospitalization or incapacitating). AEs were recorded and reported according to GCP.

\section{Pharmacokinetic Measurement}

The concentrations of BCQB in plasma and urine were determined by validated liquid chromatography-mass spectrometry methods, ${ }^{[20,21]}$. The lower limit of quantitation (LLOQ) of BCQB in plasma was $5 \mathrm{pg} / \mathrm{mL}$, while in urine it was $0.02 \mathrm{ng} / \mathrm{mL}$. The pharmacokinetic parameters were calculated by WinNonlin Professional software (Version 6.1, Pharsight Corporation, Mountain View, CA, USA) using non-compartmental methods. The pharmacokinetic parameters in these studies were maximum plasma concentration $\left(\mathrm{C}_{\max }\right)$, the time to $\mathrm{C}_{\max }\left(\mathrm{t}_{\max }\right)$, the minimum value of the steady-state plasma drug concentration $\left(\mathrm{C}_{\mathrm{min}, \mathrm{ss}}\right)$, elimination half-life $\left(\mathrm{t}_{1 / 2}\right)$, the area under the plasma concentration-time curve (AUC) from time 0 to time $\mathrm{t}\left(\mathrm{AUC}_{\mathrm{t}}\right)$, the AUC from time 0 to infinity $\left(\mathrm{AUC}_{\infty}\right)$, the steady-state $\mathrm{AUC}\left(\mathrm{AUC}_{\mathrm{ss}}\right)$, apparent clearance $(\mathrm{CL} / \mathrm{F})$, apparent total volume of distribution $\left(\mathrm{V}_{\mathrm{d}} / \mathrm{F}\right)$, the average steady-state concentration $\left(\mathrm{C}_{\mathrm{av}}\right)$, the degree of fluctuation $(\mathrm{DF})$, the accumulation ratio $\left(\mathrm{R}_{\mathrm{ac}}\right)$ 
and the percentage of BCQB dose recovered in urine $\left(\mathrm{f}_{\mathrm{e}} \%\right)$.

\section{Statistical Analysis}

Statistical analysis was performed using SPSS software version 11.0 (SPSS, Inc., Chicago, IL, USA). Prior to analysis, dose-dependent parameters $\left(\mathrm{C}_{\max }\right.$ and $\left.\mathrm{AUC}\right)$ were determined using natural logarithms of individual values. For the exploration of dose proportionality, the slope $\beta$ and $90 \%$ confidence intervals (CIs) obtained from the power model: $\ln \left(\mathrm{AUC}\right.$ or $\left.\mathrm{C}_{\max }\right)=\alpha+\beta \times \ln ($ dose $)$ were computed by analysis of covariance (ANCOVA). The regression coefficient was significant at level 0.1 . The pre-defined criterion was set as $(0.500,2.000),{ }^{[22]}$ and the criterion interval resulted in the value of $(0.500,1.500)$. The differences in pharmacokinetic parameters among dose groups were compared using ANOVA except for $t_{\text {max }}$ for which the non-parametric test (NPT) was used. Statistical comparisons between pharmacokinetic parameters of single and multiple doses were performed by the paired t-test (PTT), and the differences of pharmacokinetic parameters between male and female subjects were compared by the independent t-test (ITT). To determine whether steady state was reached in the multiple-dose study, the differences in $\mathrm{C}_{\text {min,ss }}$ on days 5,6 , and 7 were compared using ANOVA.

\section{Results}

\section{Study Population}

Healthy males and females $(n=98)$ participated in the FIH studies. No subject dropped out of the study. Baseline demographics of the study population are presented in table I.

\section{Single-Dose Pharmacokinetic Study}

The mean plasma concentration-time curves are shown in figure 2 , and the main pharmacokinetic parameters of $\mathrm{BCQB}$ are presented in table III. Absorption of BCQB after intranasal administration was rapid, with a median $t_{\max }$ of 8 minutes for 45,90 , and $180 \mu \mathrm{g}$ doses, and the plasma concentrations of $\mathrm{BCQB}$ decreased in a biphasic manner, with the mean $t_{1 / 2}$ of 8.5 hours across the doses.

The mean and $\mathrm{SD}$ values of $\mathrm{C}_{\max }, \mathrm{AUC}_{\mathrm{t}}$ and $\mathrm{AUC}_{\infty}$ versus dose relationships after single intranasal dosing of BCQB are presented in figure 3. Over the dose range studied, the mean $\mathrm{C}_{\max }$, $\mathrm{AUC}_{\mathrm{t}}$ and $\mathrm{AUC}_{\infty}$ increased linearly across the doses by linear regression analysis, with regression equations in figure 3 . Dose proportionality was observed $(p>0.05)$ by the ANOVA on the values of $\ln \left(\mathrm{C}_{\text {max }} /\right.$ dose $)$ and $\ln (\mathrm{AUC} /$ dose $)$ among the three dose groups. Moreover, the mean slopes of the plots for $\ln \left(\mathrm{C}_{\max }\right)$ or $\ln$ (AUC) versus $\ln$ (dose) were all close to 1 , and the $90 \%$ CIs of the slopes were completely contained within the predefined range $(0.500,1.500)$ for dose proportionality. The mean slopes (90\% CIs) were 1.067 (0.834, $1.300)$ for $\mathrm{C}_{\max }, 1.207(0.921,1.494)$ for $\mathrm{AUC}_{\mathrm{t}}$, and $1.051(0.762,1.341)$ for $\mathrm{AUC}_{\infty}$. Thus, $\mathrm{C}_{\max }$ and AUC proved to be dose proportional across the studied doses by different methods. The values of $t_{\max }, t_{1 / 2}, C L / F$ and $f_{e} \%$ were independent of dose $(p>0.05)$. There was no clinically significant pharmacokinetic difference ( $p>0.05$, by ITT) between males and females in the single-dose study.

\section{Multiple-Dose Pharmacokinetic Study}

The mean plasma concentration-time curves of BCQB after the first dose (day 1) and the last dose (day 7) are presented in figure 4, and the pharmacokinetic parameters from the non-compartmental analysis of measured plasma concentrations on day 1 and day 7 are provided in table IV.

No significant difference in $\mathrm{C}_{\text {min,ss }}$ was found by ANOVA analysis, indicating that steady-state conditions were achieved by day 5 after two consecutive three times daily $120 \mu \mathrm{g}$ doses of BCQB. Under steady-state conditions, BCQB was rapidly absorbed with the median $t_{\max }$ of 8 minutes and a mean $\mathrm{C}_{\max }$ of $158.3 \mathrm{pg} / \mathrm{mL}$, which were identical to the single-dose parameters (day 1). $\mathrm{BCQB}$ cleared from plasma in a biphasic manner with no significant difference of $t_{1 / 2}$ between the first and the last dose. However, the mean AUC values were higher in the multiple-dosing regimen than the corresponding values obtained after singledose (day 1) administration $(\mathrm{p}<0.01)$, and slight 

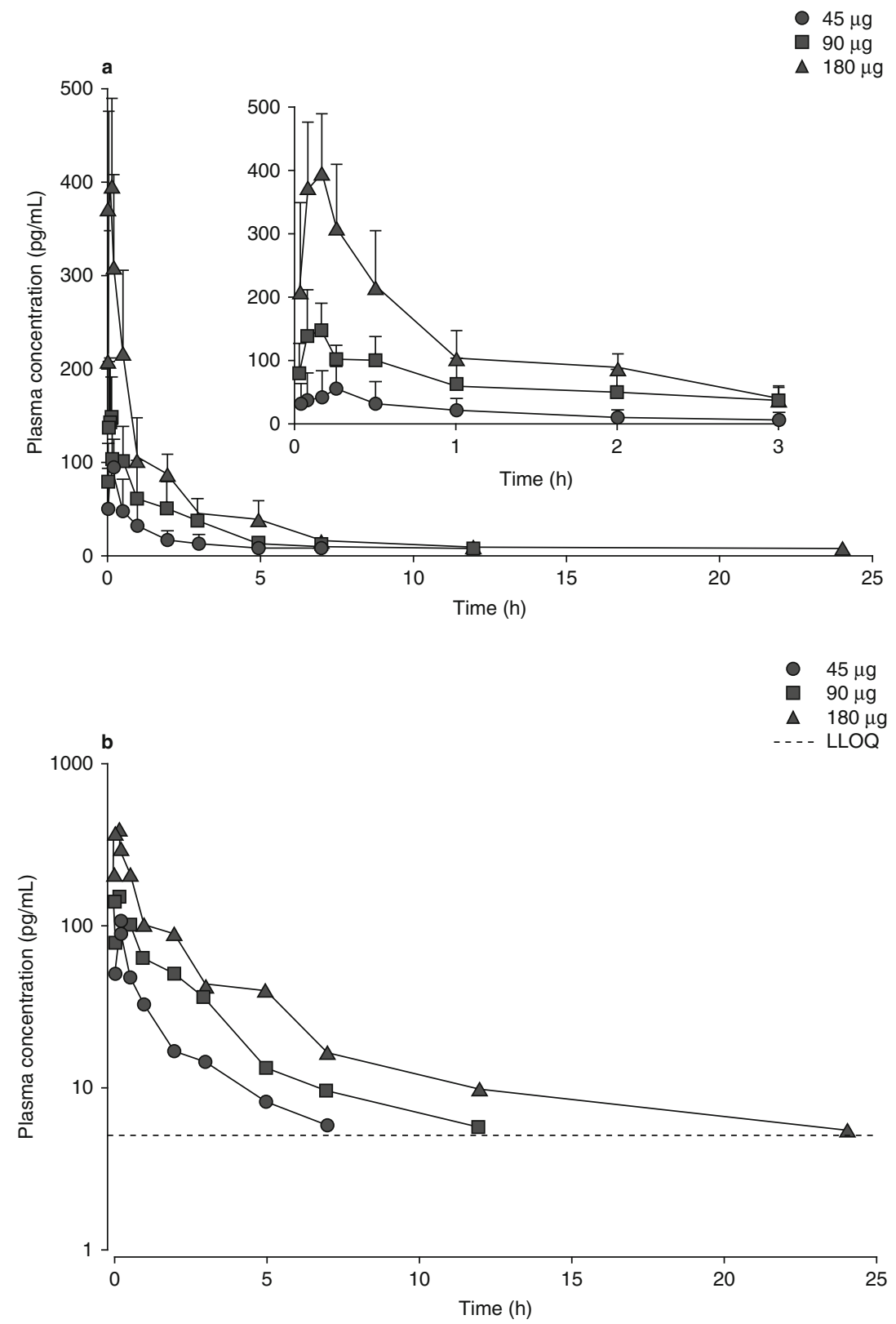

Fig. 2. Mean plasma (a) and log-scaled mean plasma (b) concentration-time profiles of bencycloquidium bromide following single intranasal doses in healthy Chinese subjects. The inset expands the first 3 hours of the profile. Data are presented as mean $+S D(n=10$ per dose). LLOQ $=$ lower limit of quantitation.

accumulation was found following repeat dosing of $\mathrm{BCQB}$ with $\mathrm{R}_{\mathrm{ac}}$ of 1.26 for $\mathrm{AUC}_{\tau}$ ( $\tau=5$ hours). A high DF of BCQB in plasma was achieved at
2.7 ( $\tau=5$ hours). Sex difference had no significant influence on $\mathrm{AUC}, \mathrm{C}_{\max }, \mathrm{t}_{\max }$, and $\mathrm{t}_{1 / 2}$ between the first and the last dose. 
Table III. Main pharmacokinetic parameters of bencycloquidium bromide in healthy Chinese subjects after single intranasal doses 45 , 90 , and $180 \mu \mathrm{g}^{\mathrm{a}}$

\begin{tabular}{|c|c|c|c|}
\hline \multirow[t]{2}{*}{ Parameters } & \multicolumn{3}{|l|}{ Dose $(\mu \mathrm{g})$} \\
\hline & $45(n=10)$ & $90(n=10)$ & $180(n=10)$ \\
\hline$\overline{C_{\max }(p g / m L)}$ & $110.4 \pm 52.5$ & 170.6 & $432.1 \pm 93.9$ \\
\hline$t_{\max }(\min )$ & $8(2-15)$ & $8(5-30)$ & $8(5-15)$ \\
\hline$t_{1 / 2}(h)$ & $7.4 \pm 5.5$ & $7.5 \pm 6.8$ & $10.7 \pm 5.3$ \\
\hline$A \cup C_{t}(n g \bullet h / L)$ & $159.5 \pm 104.6$ & $338.6 \pm 174.6$ & $743.0 \pm 264.0$ \\
\hline$A \cup C_{\infty}(n g \bullet h / L)$ & $218.6 \pm 137.6$ & $415.7 \pm 258.2$ & $831.3 \pm 291.4$ \\
\hline $\mathrm{CL} / \mathrm{F}(\mathrm{L} / \mathrm{h})$ & $298.3 \pm 228.3$ & $285.8 \pm 139.1$ & $243.8 \pm 90.2$ \\
\hline $\mathrm{Vd} / \mathrm{F}(\mathrm{L})$ & $2218 \pm 863$ & $2161 \pm 1097$ & $3438 \pm 1202$ \\
\hline$f_{e} \%$ & $4.5 \pm 2.3$ & $4.6 \pm 2.1$ & $3.6 \pm 1.6$ \\
\hline \multicolumn{4}{|c|}{$\begin{array}{l}\text { a Data are presented as mean } \pm S D \text { except for } t_{\max } \text {, which was } \\
\text { expressed as median (range). }\end{array}$} \\
\hline \multicolumn{4}{|c|}{$\begin{array}{l}\text { AUC }_{\infty}=\text { area under the concentration-time curve from time } 0 \text { to } \\
\text { infinity; } \mathbf{A} \mathbf{C C}_{\mathbf{t}}=\mathrm{AUC} \text { from time } 0 \text { to time } t \mathbf{C L} / \mathbf{F}=\text { apparent total body } \\
\text { clearance; } \mathbf{C}_{\max }=\text { maximum concentration; } \mathbf{f}_{\mathbf{e}} \%=\text { the cumulative } \\
\text { percentage of bencycloquidium bromide excreted in urine; } \mathbf{t}_{1 / 2}=\text { half- } \\
\text { life; } \mathbf{t}_{\max }=\text { time to } C_{\max } ; \mathbf{V d} / \mathbf{F}=\text { apparent volume of distribution. }\end{array}$} \\
\hline
\end{tabular}

\section{Safety and Tolerability}

BCQB was safe and well tolerated when administered as a single dose up to $450 \mu \mathrm{g}$ and multiple doses up to $150 \mu \mathrm{g}$ three times daily $(450 \mu \mathrm{g} /$ day $)$ for 15 days. No death or serious adverse events (SAEs) were reported during the study and all subjects were in good compliance. No notable mean change from baseline was recorded in the vital signs or clinical laboratory variables. No individual participant value outside the laboratory reference ranges was considered to be clinically significant, and no clinically significant change in ECG and heart rate was reported in any participant during the study.

Most subjects reported one or more AE. AEs that occurred in two or more subjects, classified according to the Medical Dictionary for Regulatory Activities system organ class and preferred terms, are listed in table $\mathrm{V}$. The most frequently reported AEs were nasal irritation (including nasal congestion, nasal dryness, redness of nasal mucosa, and epistaxis) and mydriasis. However, the nasal irritation was mild, of limited duration and no inflammation was seen on early or followup nasal examinations, while mydriasis was also mild, of limited duration and of no clinical significance. Overall, all the AEs reported were mild in intensity, expected, based on the known activity of the drug or the intranasal route of administration, and not considered to be clinically significant. There was no trend for increasing AEs with increasing doses over the dose range evaluated.
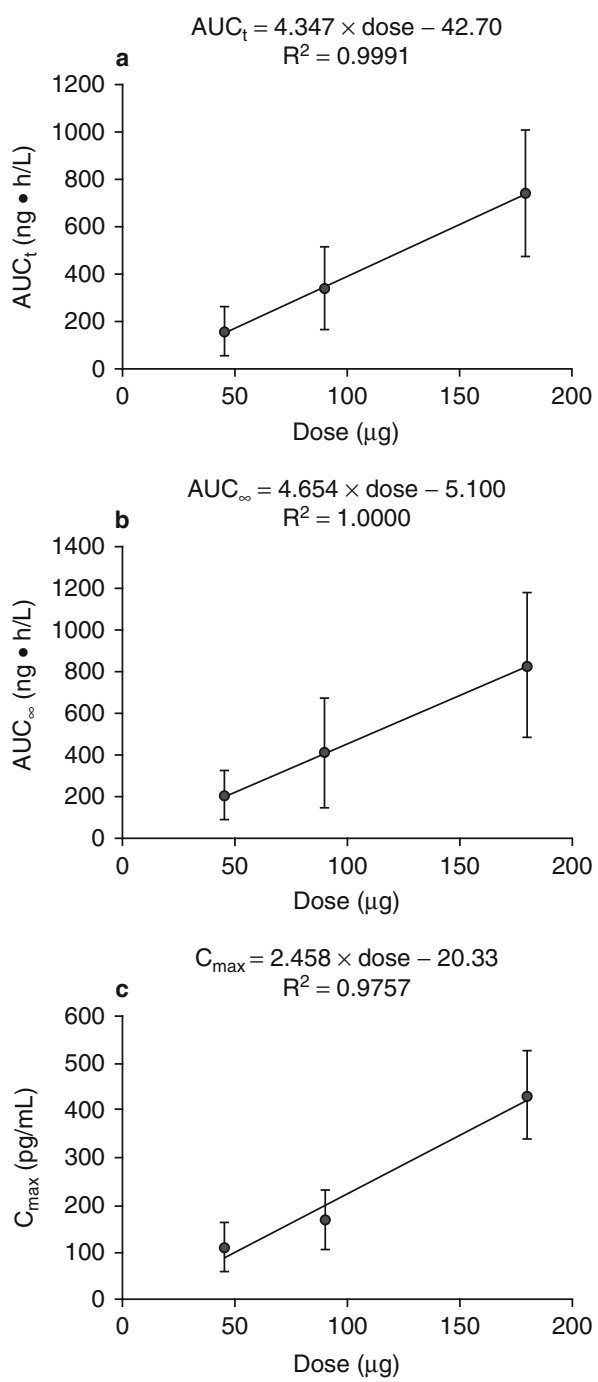

Fig. 3. Mean value $( \pm S D$ ) dose profiles of bencycloquidium bromide (BCQB) following single intranasal doses of BCQB 45, 90, and $180 \mu \mathrm{g}\left(\mathrm{n}=10\right.$ per dose). (a) $\mathrm{AUC}_{\mathrm{t}}$; (b) $\mathrm{AUC}_{\infty}$; (c) $\mathrm{C}_{\max }$. Linear regression is shown in the figure. $\mathbf{A U C}_{\mathbf{t}}=\mathrm{AUC}$ from time 0 to time $\mathrm{t}$; $\mathbf{A U C}_{\infty}=\mathrm{AUC}$ from time 0 to infinity; $\mathbf{C}_{\max }=$ maximum concentration . 


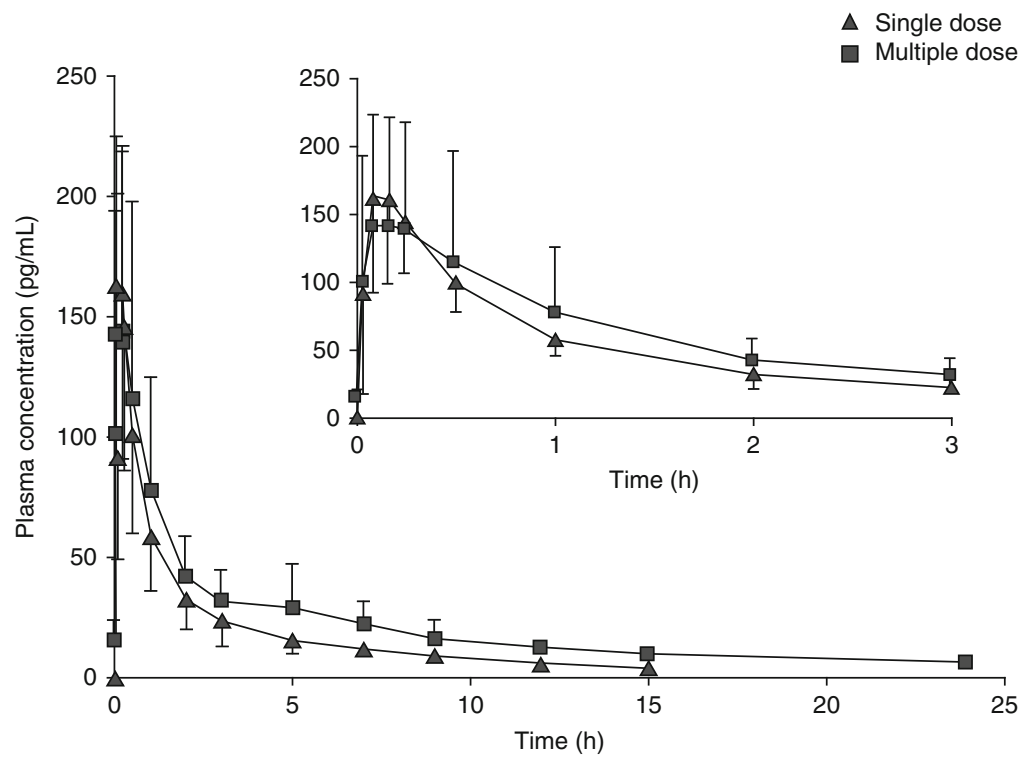

Fig. 4. Mean plasma concentration-time profiles of bencycloquidium bromide on day 1 and day 7 following multiple intranasal doses in healthy Chinese subjects, respectively. The inset expands the first 3 hours of the profile. Data are presented as mean $\pm S D(n=10$ per dose).

\section{Discussion}

At present, the anticholinergic medications used in the treatment of airway diseases are not selective for muscarinic receptor subtypes. ${ }^{[23]}$ The novel selective muscarinic M1/M3 receptor antagonists, such as aclidinium bromide ${ }^{[2]}$ and penehyclidine hydrochloride, ${ }^{[25,26]}$ are under development for the therapy of chronic obstructive pulmonary disease (COPD), while the novel agents under development for the treatment of rhinorrhea in rhinitis are limited. BCQB is under development not only for the treatment of rhinorrhea in rhinitis but also for the therapy of COPD. ${ }^{[7,11]}$ The aerosol with quantitative inhalation of bencycloquidium bromide ${ }^{[27]}$ is under development.

The objective of this FIH study was to assess the pharmacokinetics, safety and tolerability after single and multiple intranasal doses of BCQB in healthy Chinese subjects. Following single intranasal doses in healthy Chinese adult subjects, BCQB was rapidly absorbed, the plasma concentration of $\mathrm{BCQB}$ decreased in a biphasic manner, the $\mathrm{C}_{\max }$ and $\mathrm{AUC}$ of BCQB increased in proportion to the studied doses, and the mean $t_{1 / 2}$ and the mean $\mathrm{CL} / \mathrm{F}$ were independent of the administered doses. The mean $t_{1 / 2}$ of the studied dose groups ranged from 7.4 to 10.7 hours. The small difference of $t_{1 / 2}$ observed was due to assay

Table IV. Main pharmacokinetic parameters of bencycloquidium bromide in healthy Chinese subjects after multiple intranasal administration of $120 \mu \mathrm{g}$, with single administration on day 1 ; received no treatment on day 2; and continued to receive the study drug three times daily from days 3 through $7^{\text {a }}$

\begin{tabular}{lll}
\hline Parameters & Day 1 & Day 7 \\
& $120 \mu \mathrm{g}(\mathrm{n}=10)$ & $120 \mu \mathrm{g}(\mathrm{n}=10)$ \\
\hline $\mathrm{AUC}_{\mathrm{t}}(\mathrm{ng} \bullet \mathrm{h} / \mathrm{L})$ & $334.9 \pm 127.0$ & $551.4 \pm 255.4$ \\
$\mathrm{AUC}_{\infty}(\mathrm{ng} \bullet \mathrm{h} / \mathrm{L})$ & $430.0 \pm 183.6$ & $662.2 \pm 278.7$ \\
$\mathrm{t}_{\max }(\min )$ & $8(2,30)$ & $8(2,30)$ \\
$\mathrm{t}_{1 / 2}(\mathrm{~h})$ & $11.0 \pm 9.5$ & $12.3 \pm 4.2$ \\
$\mathrm{C}_{\max }(\mathrm{pg} / \mathrm{mL})$ & $175.4 \pm 77.2$ & $158.3 \pm 77.8$ \\
$\mathrm{C}_{\mathrm{ss}, \min }(\mathrm{pg} / \mathrm{mL})$ & & $16.12 \pm 8.49$ \\
$\mathrm{C}_{\mathrm{av}}(\mathrm{pg} / \mathrm{mL})$ & & $63.76 \pm 24.78$ \\
$\mathrm{DF}$ & & $2.7 \pm 0.9$ \\
$\mathrm{R}_{\mathrm{ac}}$ & & $1.26 \pm 0.39$ \\
\hline
\end{tabular}

a Data are presented as mean $\pm S D$ except for $t_{\max }$, which was expressed as median (range).

$\mathbf{A U C}_{\mathbf{t}}=$ area under the concentration-time curve from time 0 to time $\mathrm{t}$; $\mathbf{A U C}_{\infty}=\mathrm{AUC}$ from time 0 to infinity; $\mathbf{C}_{\mathrm{av}}=$ the average steady-state concentration; $\mathbf{C}_{\max }=$ maximum concentration; $\mathbf{C}_{\mathbf{s s}, \min }=$ the minimum value of the steady-state plasma drug concentration; $\mathbf{D F}=$ the degree of fluctuation; $\mathbf{R}_{\mathrm{ac}}=$ the accumulation ratio; $\mathbf{t}_{1 / 2}=$ half-life; $\mathbf{t}_{\max }=$ time to $\mathrm{C}_{\max }$. 
Table V. Treatment-emergent adverse events occurring in two or more subjects (safety population, $\mathrm{n}=58$ )

\begin{tabular}{|c|c|c|c|c|c|c|c|c|c|c|}
\hline \multirow[t]{3}{*}{ Treatment-emergent adverse events } & \multicolumn{10}{|c|}{ Safety assessment } \\
\hline & \multicolumn{7}{|c|}{ Single dose $(\mu \mathrm{g})$} & \multicolumn{3}{|c|}{ Multiple doses $(\mu \mathrm{g})$} \\
\hline & 45 & 90 & 180 & 270 & 360 & 450 & Total & 120 & 150 & Total \\
\hline $\bar{n}$ & 6 & 8 & 8 & 8 & 6 & 6 & 42 & 8 & 8 & 16 \\
\hline \multicolumn{11}{|l|}{ Upper respiratory } \\
\hline Nasal dryness & 4 & 2 & 0 & 2 & 0 & 4 & 12 & 5 & 3 & 8 \\
\hline Nasal congestion & 4 & 3 & 3 & 0 & 4 & 1 & 15 & 6 & 4 & 10 \\
\hline Epistaxis & 2 & 0 & 0 & 0 & 0 & 0 & 2 & 1 & 0 & 1 \\
\hline Color of nasal mucosa (red) & 3 & 1 & 3 & 0 & 0 & 0 & 7 & 0 & 0 & 0 \\
\hline Color of nasal mucosa (pale) & 0 & 0 & 0 & 1 & 0 & 1 & 2 & 0 & 0 & 0 \\
\hline Other nasal symptoms & 0 & 0 & 0 & 0 & 0 & 3 & 3 & 1 & 0 & 1 \\
\hline \multicolumn{11}{|l|}{ Neurology } \\
\hline Headache & 1 & 0 & 0 & 0 & 0 & 0 & 1 & 1 & 0 & 1 \\
\hline Dizziness & 2 & 1 & 0 & 0 & 0 & 0 & 3 & 0 & 0 & 0 \\
\hline Drowsiness & 0 & 0 & 0 & 0 & 0 & 0 & 0 & 2 & 0 & 2 \\
\hline \multicolumn{11}{|l|}{ Ocular } \\
\hline Ocular hypertension & 0 & 0 & 1 & 0 & 0 & 0 & 1 & 1 & 0 & 1 \\
\hline Mydriasis & 0 & 2 & 1 & 1 & 3 & 1 & 8 & 5 & 4 & 9 \\
\hline \multicolumn{11}{|l|}{ Gastrointestinal } \\
\hline Dry mouth & 0 & 0 & 0 & 2 & 0 & 0 & 2 & 0 & 0 & 0 \\
\hline
\end{tabular}

limitations in the determination of the BCQB concentration in the terminal phase of the concentration-time curve, where plasma concentrations of $\mathrm{BCQB}$ at sampling times in the latter part of the concentration-time curve were below the LLOQ $(5 \mathrm{pg} / \mathrm{mL})$ for many subjects, particularly at lower doses (figure 2). However, no statistical significance $(p>0.05)$ in $t_{1 / 2}$ was found among the studied dose groups. The duration of action of $50 \%$ of BCQB $\left(\mathrm{t}_{1 / 2}\right.$, off-set) in classical bioassays was approximately 3 hours, ${ }^{[11]}$ which was shorter than the terminal $t_{1 / 2}$ of BCQB in plasma. It may be due to the fact that the terminal $t_{1 / 2}$ in plasma is reflective of the rate of drug elimination from the body but not reflective of the duration of drug action.

In the multiple-dose study, the steady-state concentration was achieved within 3 days of consecutive dosing and the pharmacokinetic parameters of BCQB were similar to those following single dose except AUC. A slight accumulation was noted with the mean $\mathrm{R}_{\mathrm{ac}}$ of 1.26 based on $\mathrm{AUC}_{\tau}$, but the slight accumulation resulted in sustained plasma exposure upon daily dosing. A high $\mathrm{DF}$ for BCQB concentration in plasma was ob- served, for the concentrations of BCQB in plasma declined rapidly from $t_{\text {max }}$ to $\tau$.

Wide inter-subject variability in pharmacokinetic parameters was reflected in their SD (tables III and IV), but the reasons were not clear. There are several factors that can lead to the variability of pharmacokinetic parameters. First, although physicians administered BCQB carefully according to the SOPs, the intranasal administration process may cause variability. For example, while intranasal doses were administered to the lateral nasal wall, the influence of factors (such as posture, position of the head, and nasal mucosal blood flow) could increase the variability of pharmacokinetic parameters. Second, the presence of nasal mucosal physiology and pathology is another potential source of variability. ${ }^{[28]}$ For example, hyperemia would be expected to influence drug absorption after intranasal application, for the hyperemia can change the penetration of nasal mucosa, which may influence drug absorption. Third, only ten subjects had been studied for the pharmacokinetic profile in each group and the variability in one or more individual would affect 
the overall results greatly. Future clinical studies should also seek to identify the factors responsible for variability in intranasal dose delivery, deposition and mucosa absorption in order to optimize the safety profile of $\mathrm{BCQB}$ that could often be required for long-term therapy.

In this FIH study, repeated administration of BCQB did not lead to any cardiovascular adverse event in healthy subjects, consistent with previously published results in animals. ${ }^{[13,14]}$ However, future investigations to evaluate the effect of longterm doses of $\mathrm{BCQB}$ on the nasal mucosa, ECG and heart rate are warranted.

\section{Conclusion}

BCQB was safe and well tolerated in this FIH study. No SAEs occurred, no change of ECG and heart rate was observed, and all subjects were in good compliance. The mean $\mathrm{C}_{\max }$ and $\mathrm{AUC}$ of BCQB were proportional to the studied doses, and the steady state was achieved within 3 days. A slight accumulation following 5 days of $120 \mu \mathrm{g}$ three times daily dosing of $\mathrm{BCQB}$ was observed, but the elimination rate showed no change. On the basis of this study in healthy subjects, BCQB is worthy of further investigation for treating rhinorrhea in rhinitis.

\section{Acknowledgments}

This study was sponsored by Beijing Shiqiao Biological and Pharmaceutical Co. Ltd, China. Li Ding, Yongqing Wang, and Xiaoping Chen participated in the design and writing of the study protocol, and approved the final protocol. Luning Sun, Yongqing Wang, Wenjia Zhou, Weilin Sun, and Hongwen Zhang participated in the collection of data. Li Ding, Zhengyu Yan, Ning Ou, and Xiaoping Chen supported the undertaking of the study. All authors participated in the analysis and interpretation of data and in the writing of the manuscript, and approved the final manuscript. The conduct of the study, as well as opinions on analysis, conclusions and interpretation of the study data, are the responsibility of the authors. The authors take full responsibility for the content of the paper. Xiaoping Chen is employed by and is a shareholder of Beijing Shiqiao Biological and Pharmaceutical Corporation.

The authors acknowledge the contributions of Dr Jin Zhang, Mr Shailendra Shakyaand, and Mr John Kayanda Raphael for their writing assistance. This work was supported by Jiangsu province Nanjing City Innovative Graduate Research Program (no. CXZZ11 0811) and Health Bureau of Jiangsu Province (RC2011179).

\section{References}

1. Samoliński B, Sybilski AJ, Raciborski F, et al. Prevalence of rhinitis in Polish population according to the ECAP (Epidemiology of Allergic Disorders in Poland) study. Otolaryngol Pol 2009 Jul-Aug; 63 (4): 324-30

2. Wallace DV, Dykewicz MS, Bernstein DI, et al. The diagnosis and management of rhinitis: an updated practice parameter. J Allergy Clin Immunol 2008 Aug; 122 Suppl. 2: S1-84

3. Grossman J, Banov C, Boggs P, et al. Use of ipratropium bromide nasal spray in chronic treatment of nonallergic perennial rhinitis, alone and in combination with other perennial rhinitis medications. J Allergy Clin Immunol 1995 May; 95: 1123-7

4. Haddad EB, Pate H, Keeling JE, et al. Pharmacological characterization of the muscarinic receptor antagonist, glycopyrrolate, in human and guinea-pig airways. $\mathrm{Br}$ J Pharmacol 1999 May; 127: 413-20

5. Singh S, Loke YK, Furberg CD. Inhaled anticholinergics and risk of major adverse cardiovascular events in patients with chronic obstructive pulmonary disease: a systematic review and meta-analysis. JAMA 2009 Mar; 301 (12): 1227-30

6. Li J, Zhou YD, Chen XP. Experimental study on general pharmacological actions of bencycloquidium bromide. J Chongqing Med Univ 2007 May; 32: 506-10

7. Cao R, Dong XW, Jiang JX, et al. M3 muscarinic receptor antagonist bencycloquidium bromide attenuates allergic airway inflammation, hyperresponsiveness and remodeling in mice. Eur J Pharmacol 2011 Mar; 655: 83-90

8. Li J, Zhou YD, Chen XP. Curative effects of bencycloquidium bromide on allergic rhinitis in rats. Chin J New Drugs Clin Rem 2008 Mar; 27: 191-4

9. Li J, Zhou YD. Influence of bencycloquidium bromide on the nasal hypersensitivity in guinea pigs. Chin $\mathrm{J}$ Hosp Pharm 2007 Nov; 27: 1545-8

10. Li J, Zhou YD, Chen XP. Preliminary observation on the anti-inflammatory action and anti-pruritic action of bencycloquidium bromide. Chin J New Drugs 2007; 16: 1182-4

11. Jiang JX, Cao R, Deng WD, et al. Characterization of bencycloquidium bromide, a novel muscarinic M3 receptor antagonist in guinea pig airways. Eur J Pharmacol 2011 Mar; 655: 74-82

12. Li J, Zhou YD, Chen XP. Selectivity of bencycloquidium bromide to subtypes of muscarinic acetylcholine receptors. Chin J New Drugs Clin Rem 2010 Jan; 29: 45-9

13. Li J, He H, Zhou YD, et al. Subchronic toxicity and toxicokinetics of long-term intranasal administration of bencycloquidium bromide: a 91-day study in dogs. Regul Toxicol Pharmacol 2011 Nov; 59: 343-52

14. Li Z, Chen XP, Li J. Observation on toxicity of bencycloquidium bromide nasal spray in rats. China Pharm 2009 Sep; 18: 6-7

15. Xu Q, Ding L, Liu WY, et al. Determination of bencycloquidium bromide in rat plasma by liquid chromatographyelectrospray ionization-mass spectrometry. J Chromatogr B 2007 Feb; 846: 209-14

16. Xu Q, Ding L, Liu WY, et al. Determination of bencycloquidium bromide, a novel anticholinergic compound, in rats bile, urine and feces by LC-ESI-MS. Chin J Clin Pharmacol Ther 2007 Apr; 4: 385-91 
17. Xu Q, Ding L, Liu WY, et al. Determination of bencycloquidium bromide, a novel anticholinergic compound, in rat tissues by liquid chromatography-electrospray ionization mass spectrometry. Eur J Mass Spectrom 2008; 14 (5): 319-27

18. Xu Q, Ding L, Liu WY, et al. Study of the metabolites of bencycloquidium bromide racemate, a novel anticholinergic compound, in rat bile by liquid chromatography tandem mass spectrometry. Eur J Mass Spectrom 2008; 14 (2): 99-105

19. Jiang B, Ruan ZR, Lou HG, et al. Determination of bencycloquidium bromide in dog plasma by liquid chromatography with electrospray ionization tandem mass spectrometry. Biomed Chromatogr 2010 May; 24 (5): 490-6

20. Zhou WJ, Ding L, Wang YQ, et al. Solid phase extraction and liquid chromatography-electrospray ionization-mass spectrometry for the determination of bencycloquidium bromide in human plasma. J Chromatogr B 2009 Apr; 877 (10): 897-901

21. Zhou WJ, Ding L, Xu GL, et al. Determination of bencycloquidium bromide in human urine using weak cationexchange solid-phase extraction and LC-ESI-MS: method validation and application to kinetic study of urinary excretion. J Pharm Biomed Anal 2009 Aug; 50 (1): 35-40

22. Hummel J, McKendrick S, Brindley C, et al. Exploratory assessment of dose proportionality: review of current approaches and proposal for a practical criterion. Pharm Stat 2009 Jan-Mar; 8 (1): 38-49
23. Coulson FR, Fryer AD. Muscarinic acetylcholine receptors and airway diseases. Pharmacol Ther 2003 Apr; 98 (1): 59-69

24. Sentellas S, Ramos I, Albertí J. Aclidinium bromide, a new, long-acting, inhaled muscarinic antagonist: in vitro plasma inactivation and pharmacological activity of its main metabolites. Eur J Pharm Sci 2010 Mar; 39 (5): 283-90

25. Xiao HT, Liao Z, Mo ZJ. Progress in pharmacokinetics of penehyclidine hydrochloride. Chin J N Drugs 2009 Nov; 18 (10): 887-90

26. Yu Q, Xiang J, Liang MZ, et al. Determination of penehyclidine in human plasma by HPLC-MS/MS. Chin J N Drugs 2007 Nov; 18 (10): 591-3

27. Jin F, Zhao SQ, Zhang L, et al. Aerosol with quantitative inhalation of bencycloquidium bromide and preparation method thereof. CN patent 200910081661.0. 2009 Apr 8

28. Rudy AC, Coda BA, Archer SM, et al. A multiple-dose phase I study of intranasal hydromorphone hydrochloride in healthy volunteers. Anesth Analg 2004 Nov; 99 (5): 1379-86

Correspondence: Professor Li Ding and Professor Zhengyu Yan, China Pharmaceutical University, 24 Tongjiaxiang, Nanjing, 210009, China.

E-mail: dinglidl@hotmail.com 